Meta

Journal des traducteurs

Translators' Journal

\title{
Ressources néologiques du français contemporain
}

\section{Jean Darbelnet}

Volume 12, numéro 4, décembre 1967

Aspects de la terminologie

URI : https://id.erudit.org/iderudit/002470ar

DOI : https://doi.org/10.7202/002470ar

Aller au sommaire du numéro

Éditeur(s)

Les Presses de l'Université de Montréal

ISSN

0026-0452 (imprimé)

1492-1421 (numérique)

Découvrir la revue

Citer cet article

Darbelnet, J. (1967). Ressources néologiques du français contemporain. Meta, 12(4), 111-117. https://doi.org/10.7202/002470ar d'utilisation que vous pouvez consulter en ligne.

https://apropos.erudit.org/fr/usagers/politique-dutilisation/ 


\section{RESSOURCES \\ NEOLOGIQUES \\ DU FRANÇAIS \\ CONTEMPORAIN}

Dans son petit livre le Guide du bon usage ${ }^{1}$, Albert Dauzat s'est vigoureusement élevé contre ce qu'il appelait la sclérose de la dérivation française, qui risquait, disait-il, "d'ouvrir toutes grandes les portes aux anglicismes et aux formations gréco-latines dont l'abus n'est pas à démontrer ». Deux ans plus tôt, Alfred Sauvy, cité par Dauzat, avait déjà condamné "l'impuissance créatrice de la langue », la répugnance à l'égard des mots nouveaux formés avec les ressources du français, état d'esprit où il voyait un «malthusianisme linguistique [qui] non seulement ne cherche pas l'évolution mais l'empêche par tous les moyens ».

Remarquons que Dauzat semble s'attacher surtout à la dérivation et condamner les formations gréco-latines. Parallèlement, Alfred Sauvy demande des mots formés à partir du vieux fonds français. Peut-être conviendrait-il d'élargir le débat et de considérer, sans se borner à la dérivation, les ressources, quelles qu'elles soient, dont le français dispose pour enrichir son vocabulaire. Cela ne veut pas dire qu'il ne faille pas préférer en fin de compte les formations authentiquement françaises aux formations gréco-latines.

D'une façon générale, on peut dire que le français est beaucoup moins bien placé que d'autres langues pour faire des mots nouveaux. Il n'a pas, à cet égard, la souplesse des langues germaniques et, en particulier, de l'anglais et de l'allemand. De plus, il ne semble pas qu'il utilise pleinement ses ressources. Comme le signalent Albert Dauzat et Alfred Sauvy, il est victime d'une certaine timidité, de ce qu'on a justement appelé l'immobilisme linguistique.

Or, il se trouve que nous vivons une époque de transformations techniques et sociales (celles-ci dérivant de celles-là) et que le besoin de créer de nouvelles ressources lexicales pour rendre compte des nouvelles réalités est particulièrement aigu. Le français ne peut rester une langue de grande communication que s'il s'adapte aux besoins du monde d'aujourd'hui et de demain. Il n'est pas dans sa nature de posséder la plasticité d'autres langues, mais il reste possible et il est

1. Paris, Delagrave, 1954. 
nécessaire de tirer le meilleur parti des procédés dont il dispose pour se donner des mots nouveaux. Et pour cela il n'est peut-être pas inutile de les passer en revue.

Tout d'abord, rappelons qu'il n'est guère possible de créer un mot ex nihilo, c'est-à-dire sans s'appuyer sur quelque chose qui existe déjà. On a cru un moment que le mot $g a z$ était une exception. En fait Van Helmont l'a créé à partir du mot grec qui veut dire chaos.

La façon la plus simple de former un mot est sans doute l'onomatopée, le mot évoquant le bruit de la chose qu'il désigne. C'est le cas de frou-frou, coucou, pépier. Mais ce procédé donne des mots imagés, pittoresques, plutôt que les termes techniques dont nous avons surtout besoin à l'heure actuelle.

Très simple également est le moyen qui consiste à utiliser les noms propres. La chose nouvelle reçoit ainsi le nom de son inventeur ou du pays où elle a pris naissance. Nous avons eu ainsi berline, landau, poubelle, carter, etc. Le vocabulaire médical comprend des noms de maladies qui sont ceux des savants qui les ont identifiées: la maladie de Parkinson, le mal de Pott, etc.

En troisième lieu, il y a toujours la ressource d'emprunter, et l'on sait que, depuis le XviIr siècle, c'est surtout à l'anglais que le français emprunte. La facilité même de ce procédé explique l'abus auquel il donne lieu. L'emprunt prend plusieurs formes, qu'il ne saurait être question d'examiner ici, car le sujet ne se laisse pas traiter en quelques lignes, et d'ailleurs ce procédé, par sa simplicité même, est en marge de ceux auxquels il convient de s'arrêter et qui sont ceux par lesquels la langue utilise son propre fonds.

Il est commode de ranger ces procédés sous le terme général de provignement, qui recouvre ainsi ce que les grammaires appellent composition et dérivation. De plus, il y a également le provignement sémantique, par lequel un mot reçoit de nouvelles acceptions.

Le français pratique moins la composition que les langues germaniques. Au début de son histoire, il a composé des mots dans lesquels le déterminant précédait le déterminé; mais assez tôt il a préféré l'ordre inverse et le type vinaigre a pris le pas sur le type sangsue. Nous avons ainsi cerf-volant à côté de chauve-souris. Cependant, la séquence régressive, c'est-à-dire celle qui met le déterminant avant le déterminé, est loin d'être abandonnée; on la retrouve dans le vocabulaire technique, surtout avec les formations gréco-latines: téléphone, thermodynamique, omnipraticien, motoculture. Les mots composés avec le préfixe auto-, et ils sont de plus en plus nombreux, appartiennent à cette catégorie. Et nous disons nordafricain, sud-américain à côté de Afrique du Nord et Amérique du Sud. À ce propos on peut craindre que «Nord Viêt-nam» ne gagne sur Viêt-nam du Nord, séquence plus naturelle en français et qui nous a donné, sans que l'usage hésite, Irlande du Nord.

L'une des ressources les plus importantes du français dans le domaine de la composition est celle qui lui permet de faire suivre un verbe à la troisième personne du singulier d'un complément direct: essuie-mains, abat-jour, chauffe-bain. On remarque que la séquence dans ce cas est progressive conformément aux ten- 
dances de la langue, puisque le déterminant (c'est-à-dire le complément direct) vient après le déterminé (le verbe). Mais il existe un procédé encore plus souple, c'est celui de la juxtaposition. Cette fois encore, le déterminant suit le déterminé, avec cette différence que ce ne sont plus les verbes et leurs compléments qui sont ainsi combinés, mais des substantifs comme dans timbre-poste ou des participes passés et des noms comme cousu main. On remarquera que, par rapport à la construction syntaxique normale, ce genre de composé, qui ne comporte pas nécessairement un trait d'union, suppose des mots sous-entendus: timbre pour la poste, cousu à la main. La langue technique fait un large usage de ce procédé: les messages météo, une voiture tous terrains, la position «couchette» d'un siège d'auto, attendre sur le quai direction Auteuil, les allocations-logement, l'assurance-maladie, etc.

À cette catégorie on peut rattacher, compte tenu de certaines différences, des juxtapositions de mots à séquence tantôt régressive tantôt progressive et qui servent souvent à désigner des raisons sociales: France-Presse, Air-France, Air Canada. Quelquefois il y a télescopage: Eurasie, Eurafrique, Eurailpass (carte de circulation sur les chemins de fer européens) et franglais.

Par ailleurs, il y aurait lieu de considérer comme composés certaines combinaisons de mots qui sont devenues habituelles. Bien que ces mots ne paraissent pas à première vue étroitement unis cntre eux, leur unité de sens, le fait qu'ils correspondent parfois à un seul mot soit en français (avec impunité = impunément) soit dans une autre langue (clouer au pilori $=$ to pillory) leur assure une cohésion analogue à celle des composés que les dictionnaires donnent à leur place alphabétique, tout comme des mots simples. On peut remarquer également qu'il existe entre ces unités des différences paronymiques analogues à celles qui opposent des mots simples. Il convient de distinguer mettre à jour et mettre au jour tout comme on doit se garder de confondre percepteur et précepteur. Et ainsi, il n'y a aucune raison de ne pas compter, parmi les néologismes des vingt dernières années, des syntagmes tels que cartes perforées, bandes dessinées, le tout compris, le prêt à porter, les centres d'intérêt, les remontées mécaniques, les deux-roues, etc.

D'autre part, notre langue peut se créer des unités lexicales nouvelles en faisant passer un mot d'une partie du discours dans une autre. Le jour où l'on a employé l'adjectif automatique comme substantif à propos du téléphone (ex.: ces localités sont maintenant dotées de l'automatique), on a en fait créé un mot nouveau. Parmi les termes ainsi créés, on peut citer les enseignants, les tranquillisants, la didactique, la problématique. Il n'y a aucune raison de mettre en doute la validité de ces deux derniers néologismes, puisque nous trouvons tout naturel de dire la poétique, la physique, la symbolique. Il ne s'agit plus ici de composition, mais de dérivation, et plus exactement de dérivation impropre.

La dérivation proprement dite est souvent restreinte aux mo's formés par suffixation. Mais rien n'empêche d'y comprendre aussi des mots à préfixe. Du point de vue auquel nous nous plaçons, il est commode de ne pas établir de différence de nature entre la suffixation et la préfixation. Ce qui nous intéresse, c'est de voir quels sont les suffixes et les préfixes qui sont les plus vivants et par conséquent les plus utiles. 
Il y a cinquante ans le suffixe -thèque n'entrait guère que dans le mot bibliothèque et dans le germanisme pinacothèque, qui désigne un célèbre musée de Munich. Aujourd'hui, il y a toute une série de mots en -thèque: cinémathèque, discothèque, phonothèque, cartothèque. Mais l'usage de ce suffixe est forcément restreint, le nombre des choses ainsi constituées en collections ne pouvant être illimité. Plus fréquent est le recours aux suffixes -ner et -nel ajoutés à des substantifs en -tion. Les puristes ont protesté contre la formation de solutionner, ovationner, réceptionner, ambitionner, révolutionner, mais il n'empêche que ces verbes sont souvent plus commodes à employer que ceux qu'ils tendent à évincer et ils apportent quelquefois une nuance utile, comme par exemple celle qui sépare réceptionner de recevoir. Les adjectifs en -nel sont appelés à se développer. Sans doute éducationnel est-il dû à l'influence de l'anglais educational, mais nous en aurons vraisemblablement besoin. Fonctionnel permet d'exprimer une idée qui autrement aurait du mal à percer. Et si les militaires se sont annexé opérationnel, c'est parce qu'il leur permet de distinguer entre les opérations et l'instruction, par exemple. Ainsi des unités de la marine peuvent être divisées en deux groupes, l'un opérationnel et l'autre dit d'instruction, et apparemment on n'exprime pas tout à fait la même chose quand on dit qu'un certain matériel de guerre approche du stade opérationnel au lieu de dire qu'il va être mis en service.

Les suffixes -iste et -able restent parmi les plus employés. Avec -iste on peut former des noms d'adhérents ou de partisans (les naturalistes, les calvinistes, les benbellistes) ou désigner ceux qui exercent certaines professions (fleuristes, latinistes, pompistes, secouristes, documentalistes). Les adjectifs formés avec le suffixe -able peuvent devenir des noms: un imperméable, un contribuable, nos honorables, le coupable, les notables. Tout récemment la profession médicale a adopté le mot jetable pour désigner ce qui ne doit servir qu'une fois, ce qu'il faut jeter après un seul usage, et il y a maintenant dans le matériel médical la catégorie des jetables. Ce terme surprend au premier abord, mais il n'y a pas de raison pour qu'on ne puisse pas faire avec jetable ce qu'on a fait avec coupable.

On a souvent critiqué l'emploi au pluriel des substantifs en -té: responsabilités, activités, etc. On allègue que le gouvernement, par exemple, ne saurait prendre «ses responsabilités», sa responsabilité étant une et non multiple. Ce point de vue se défend logiquement, mais il ne tient pas compte d'un fait de langue qui remonte loin et qui existait déjà en latin, à savoir que les mots abstraits peuvent se mettre au pluriel pour désigner des aspects concrets de la qualité abstraite qu'ils expriment au singulier. La possibilité est la qualité de ce qui est possible, les possibilités dont je dis qu'il y en a plusieurs sont des cas concrets du possible, des exemples de possibilité. Nous allons voir les curiosités d'un pays que nous visitons, nous entrons dans un magasin de nouveautés et nous parlons des activités de quelqu'un dont l'activité serait simplement le fait d'être actif dans telle ou telle situation. Comparez: "Il a déployé une activité fébrile» et « la police enquête sur ses activités ».

Nous vivons à l'époque des initiales qui permettent d'abréger les désignations. Les sigles sont des mots et ce qui le prouve, c'est qu'ils peuvent former des dérivés. De C.G.T. (Confédération générale du travail, mais non, dans ce cas, 
Compagnie générale transatlantique) on a fait cégétiste, et on a lancé onusien, à partir de l'O.N.U., par exemple à propos de timbres: les timbres onusiens. Mais le cas le plus curieux est celui de igamie qui signifie en France la circonscription d'un igame, igame étant la forme concentrée de I.G.A.M.E., inspecteur général de l'administration en mission extraordinaire. Les igames s'appellent aussi superpréfets ou préfets de région.

Le suffixe -iser forme de nombreux verbes à partir de substantifs ou d'adjectifs. À leur tour, ces verbes donnent naissance à des noms par adjonction du suffixe -isation au radical. De plus, les mots de ces deux séries ont des contraires formés au moyen des préfixes dé- ou in-. Ainsi nous avons défanatiser et défanatisation, dépersonnaliser et dépersonnalisation, ces derniers termes pouvant s'appliquer à des troubles psychiques, à la faculté de dédoublement et aussi à un état social où la personne ne compte plus. André Siegfried avait même risqué le néologisme démercatoriser en faisant allusion à la projection de Mercator: « Il nous faut nous "démercatoriser », c'est-à-dire nous habituer à considérer toujours que la Terre est ronde. »Dans le même ordre d'idée, on se rappelle que la Russie est passée, il y a quelques années, par une phase de déstalinisation. Il existe une série parallèle avec les suffixes -ifier et -ification: dénazifier, dénazification.

Parmi les suffixes les mieux accordés à notre époque on remarque auto-, pré-, et para-. La fréquence du préfixe auto- est naturelle à une époque où un nombre croissant de choses se font automatiquement. Selon les secteurs de notre activité, on nous parle d'autofinancement, d'autodéfense, d'autocensure, d'autocritique, d'autocuiseur, d'auto-information (il paraît que c'est le devoir d'un citoyen soucieux de la chose publique), d'automédication. Et la liste est loin d'être complète. On remarquera que le scrupule qui retenait nos pères de combiner un préfixe grec avec un mot d'origine latine a disparu devant la nécessité d'exprimer des notions nouvelles en nombre croissant. Naturellement, parce qu'elle est facile, la préfixation de auto- donne lieu à des abus. Est-il bien nécessaire de parler d' "autosatisfaction » (peut-être sous l'influence de self-satisfaction) alors que nous avons déjà complaisance ? Par ailleurs, le général de Gaulle a créé, vers 1960, à propos de la guerre d'Algérie, le mot autodétermination. La constitution de la $\mathrm{V}^{e}$ République se contente de libre détermination. Avant 1914 on appelait cela «le droit des peuples à disposer d'eux-mêmes », ce qui était plus long, mais aussi plus clair.

Certains suffixes sont tombés en désuétude. Nous ne formons plus guère de mots en mal-. Et à ce propos, il serait intéressant de comparer un dictionnaire de 1920 et un dictionnaire de 1967 en ce qui concerne les mots commençant par autoet les mots commençant par mal-. La liste s'est allongée dans le premier cas et raccourcie dans le second. Cependant il y a parfois des reviviscences. C'est ainsi qu'il a été question en 1958 du malconfort des libéraux, peut-être parce que Camus avait précédemment employé ce mot dans la Chute. Mais c'est là un de ces usages figurés auxquels les mots désuets se prêtent encore. Plus frappant est l'emploi de malentendant comme terme technique et atténuatif de sourd: «Équipement des salles à l'intention des malentendants ». Par ailleurs les spécialistes continuent à parler de malnutrition, qu'il ne faut pas confondre avec sous-alimentation.

Le préfixe pré- est ancien, préavis remontant à la fin du XIVe siècle. L'évolution 
de nos techniques rend ce préfixe très utile pour marquer certaines phases de fabrication. Les archéologues parlent depuis assez longtemps de l'Amérique précolombienne, mais ce n'est que depuis la dernière guerre que nous avons préfabriqué, préfinancement, et le recul du temps nous amène à considérer les époques préindustrielles et préatomiques. Les réclames des commerçants nous parlent de viande préemballée et même de «précuisine».

Para- qui nous est venu dans des mots d'origine grecque et latine, a servi et sert encore à former des composés français où il marque l'idée de protection. Parapluie est du XVII ${ }^{\mathrm{e}}$ siècle et paratonnerre remonte à 1779. Plus récemment, ce préfixe permet de former des mots où il ajoute l'idée de périphérie. Les formations paramilitaires ne sont pas destinées à combattre l'armée, elles s'apparentent à l'armée. On reconnaît maintenant des professions parajudiciaires (notaires, huissiers, etc.) et paramédicales (infirmières), et il existe une parafiscalité destinée à financer certaines œuvres d'assistance sociale.

Et enfin il y a toujours la possibilité de créer des unités lexicales sans créer de mots nouveaux en ajoutant un sens nouveau à un mot déjà existant. C'est ce qu'on peut appeler le provignement sémantique. Ce mode d'enrichissement de la langue a toujours existé. En consultant un dictionnaire, nous avons l'impression que les mots ont toujours eu les sens qu'ils ont maintenant. En fait, leur contenu sémantique s'est constitué graduellement au fur et à mesure des besoins d'expression. Il suffit de chercher dans un dictionnaire récent et dans un dictionnaire d'il y a quarante ans les mots satellite, secteur et communauté, pour se rendre compte de la façon dont les mots s'introduisent dans des domaines où ils ne jouaient jusque-là aucun rôle.

Certaines de ces acceptions nouvelles viennent de l'anglais. C'est le cas avec réaliser au sens de se rendre compte et avec contrôler au sens de diriger. Il est difficile de ne pas accepter le nouveau sens commercial de promotion (promotion des ventes), et procédure risque fort de prendre tous les sens de procéder au lieu de se limiter au sens judiciaire. Par contre, réadaptation et rééducation sont assez bien installés dans l'usage pour que réhabilitation reste cantonné dans son acception judiciaire.

L'utilisation des ressources néologiques du français d'aujourd'hui se heurte souvent à l'opposition des usagers. Cette opposition n'est pas toujours justifiée, ce qui lui vaut les critiques d'Albert Dauzat et d'Alfred Sauvy citées au début de cet article.

Il est évident qu'on ne doit pas accepter n'importe quoi, mais il existe un critère facile à invoquer, c'est celui des précédents. Si un mot nouveau se révèle nécessaire et si sa formation inscrit ce mot dans une série déjà existante, on ne voit pas pourquoi il ne serait pas admis dans le bon usage. Ainsi on ne peut guère s'opposer à réservation qui est parfaitement formé et qui est à réserver ce que observation est à observer ou innovation à innover. Sans doute continuera-t-on à dire: retenir une place, retenir une table, retenir une chambre, mais l'employé d'un grand hôtel qui reçoit les demandes des clients ne pourra guère, au téléphone, répondre autre chose que «Service des réservations ». 
De la même façon, il n'y a pas lieu de condamner systématiquement certains verbes en -er formés à partir des substantifs de même sens. Jusqu'à nouvel ordre, on a le droit de préférer faire escale à «escaler», verbe qui a figuré sur un avis de la Transat à propos du France. Mais la vogue du ski semble autoriser le verbe skier, encore que faire du ski paraisse plus conforme à la démarche du français. Dans un numéro de l'Express d'octobre 1964, on trouve «tourner» au sens de faire une tournée: «Cet orchestre tournera en Allemagne. Il s'agit ici d'une adjonction de sens à partir du substantif tournée. Cette adjonction est possible, mais elle se heurte à une ambiguïté à cause de la multiplicité de sens que possède déjà le verbe tourner. De ce point de vue elle n'est pas désirable.

Passant dans un autre secteur de provignement, nous pouvons dire que la coopération entre la France et d'autres pays francophones est devenue suffisamment importante pour que ceux qui servent aux fins de cette coopération s'appellent des coopérants ${ }^{2}$. Coopérant est aussi valable que enseignants et tranquillisants.

On a vu par l'échantillonnage présenté ci-dessus, que tout en n'ayant pas la plasticité d'autres langues le français possède quand même des moyens de s'enrichir qui ne sont pas négligeables. Mais il est nécessaire que dans la situation où il se trouve actuellement, ces moyens soient utilisés dans toute la mesure du possible. On estime avec raison qu'il est menacé par l'anglicisation. Mais trop souvent ceux qui pensent ainsi acceptent difficilement les mots nouveaux formés à partir de mots authentiquement français. Il est à peine besoin de souligner ce qu'une telle attitude a de contradictoire. Il est incontestable que nous continuerons à avoir besoin de mots nouveaux et qu'il faudra ou créer ou emprunter. Pour ne pas trop emprunter (il y aura toujours des emprunts), il nous faut faire l'inventaire de nos ressources néologiques et de leurs possibilités et ne pas hésiter à les utiliser en s'inspirant des types de formation déjà existants.

JEAN DARBELNET

2. Cf. le Monde, Sélection hebdomadaire, semaine du 10 au 16 août 1967.

Vol. $12-\mathrm{N}^{\circ} 4$
Décentbre 1967 